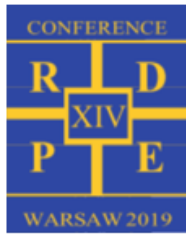

\title{
New blade tip-timing system for measuring rotor blade vibration of steam and gas turbines
}

\author{
Piotr K owaleczko ${ }^{2}$,Romuald Rządkowski ${ }^{1,2^{*}}$, Leszek K ubitz ${ }^{1}$, Paweł Troka ${ }^{1}$, Paweł K owaleczko ${ }^{2}$, Ryszard \\ Szczepanik ${ }^{2}$ \\ ${ }^{1}$ Institute of Fluid-Flow M achinery Polish A cademy of Sciences, ul. Józefa Fiszera 14, 80-231 Gdańsk, Poland, z3@ imp.gda.pl \\ ${ }^{2}$ A ir Force Institute of Technology, ul. Księcia Bolesława 6, 01-494 W arsaw, Poland, piotr.kowaleczko@itwl.pl
}

\begin{abstract}
One of the crucial issues regarding turbine maintenance is registering blade vibrations. These vibrations can cause serious damage to the engine. Turbine blade vibrations were measured during nominal speed as well as during run up and run down. A new, low cost Blade Tip Timing (BTT) is presented in this paper. It composes of two main modules: the FPGA unit and PC unit. The system is based on the TerasIC DE0-CV development board controlled by the Cyclone V 5CEBA 4F23C 7 chip. Units communicate via an Ethernet interface. The system measures a signal for every revolution as well as up to three signals coming from independent rotor blade sensors. The PC unit records these data in .csv files. The system can be adapted to process the signals of additional sensors. The measurements of the $1^{\text {st }}$ stage compressor blade vibrations in an SO-3 engine prove that the system works correctly, with no data loss during transmission between system units, and compares well with other measurement systems as well as numerical results.
\end{abstract}

\section{Introduction}

Blade Tip Timing (BTT) is one of the methods of measuring blade vibrations.

In the industry, each company has its own tip-timing system [1] [2] [3] [4] [5]. These system consist of electronic hardware to measure the time of blade arrival and the signal at every rotor revolution. The LabVIEW was used in [6]. Electronic systems are generally very expensive.

In this paper, for the first time, a very cheap system will be presented. The measurements of this system for rotor blade amplitudes in the $1^{\text {st }}$ stage of an SO-3 engine compare well with the measurements of other systems and numerical results.

\section{Blade tip-timing measurement system}

The current configuration of the proposed BTT system is the third version of the initial concept, which was originally designed for low-speed steam engine turbines only. In the first version of the system, the FPGA and PC unit communicated via an RS-232 serial connection. As the basic non-functional requirement for the system was its low cost, the FPGA platform based on a TerasIC DE1 board [7] with a Cyclone II device [8] were selected. System operation was stable and the error rate was at acceptable level. Notwithstanding this, due to the low RS232 connection speed, data acquisition was only possible for low turbine rotation frequencies (up to $50 \mathrm{~Hz}$ ) and only one blade sensor. Hence, a second version of the system was developed. The acquired data from four sensors were stored on SD cards. Only after PC processing could this data be read. It was also impossible to save all the data (even from a single turbine sensor), because of the limitations of the SPI mode in SD cards. As the speed test (monitored with an oscilloscope) have shown, data integrity was highly dependent on the SD cards. Multiple data packets were lost because of the prolonged time it took for SD cards to record the data. The error rate was not acceptable even for turbine rotation frequencies lower than $50 \mathrm{~Hz}$. Hence, it was impossible for the second version of the system to meet jet engine blade vibration requirements, such as: the maximum turbine rotation frequency $-250 \mathrm{~Hz}$, the number of turbine blades -75 and the number of blades' sensors -3 .

The $3^{\text {rd }}$ version of the system was designed to meet the above requirements and also allow for these parameters to be altered. The limitations of the $1^{\text {st }}$ and $2^{\text {nd }}$ versions did not result from FPGA architecture, but the way in which the data were transmitted. Therefore, the $3^{\text {rd }}$ version uses the Ethernet standard for communication between the FPGA and PC unit, supporting data transmission speeds up to $100 \mathrm{~Gb} / \mathrm{s}$. The $3^{\text {rd }}$ version of the system composes of two main parts of communicating via UDP protocol: a FPGA-based unit and a receiver desktop application called the PC unit. Both modules have been developed for the first time using commercial off-the-shelf (COTS) hardware components.

\subsection{FPGA unit}

The measurement component of the system is based on the TerasIC DE0-CV development board [9]. The core of the hardware is the Cyclone V 5CEBA4F23C7 FPGA device [10]. The platform is fed by a $50 \mathrm{MHz}$ clock signal, which is downscaled in the system to $10 \mathrm{MHz}-$ the 
resolution used during measurements. The software has been developed using Quartus Prime 18.1.0, with both IP Core functionalities provided by the IDE [11] and with modules manually programmed in Verilog and VHDL. The Quartus project consists of 6 types of software modules, described below.

\subsubsection{Time of Arrival measurement modules}

The system allows for the measurement of signals from one 'once per revolution' sensor and 3 independent blade sensors. In other words, four Time of Arrival (ToA) measurement modules have been developed in the system. Each of them measures rising edge impulse ToA with 10 $\mathrm{MHz}$ precision. The system's internal main clock has 64bit architecture. This solution allows for measurements to be unaffected by overflow errors (time to first error $>58 \mathrm{k}$ years). The minimal turbine revolution frequency in the current version should be greater than $0.03 \mathrm{~Hz}$ in order to guarantee error-free ToA acquisition. This applies only if impulses coming from blade sensors are of a clear quadratic shape. This limitation results from system's architecture and it can be easily changed if necessary. The upper frequency limit depends on the number of rotor blades in the turbine and on 10Base-T standard implementation in the Ethernet module. The system was originally designed for monitoring turbines with 75 blades and rotation speeds of between $10 \mathrm{~Hz}$ to $250 \mathrm{~Hz}$. Laboratory tests have shown that the system easily delivers error-free ToA values for 75 blades with a rotation frequency of even $500 \mathrm{~Hz}$. The theoretical maximum frequency allowed by the system for 75-blade turbine results from 10Base-T standard's limitations and is equal to ca. $1100 \mathrm{~Hz}$.

\subsubsection{Data assembling modules}

Quartus Prime project includes two modules responsible for the preparation of acquired data to be sent. ToA values for each of the 4 sensors are uniquely marked (in order to be distinguished at a later stage) and stored in a FIFO buffer (IP Core implementation).

\subsubsection{Data sending modules}

The system is compatible with the 10Base-T Ethernet standard. Once per revolution, ToA values acquired for four sensors are sent to the PC unit together with an unique revolution number. The UDP protocol has been implemented for the Ethernet connection.

\subsubsection{System clock module}

In order to guarantee $10 \mathrm{MHz}$ resolution of measurements, a PLL module has been implemented in the project. An IP Core component was used. The base clock signal is provided by a $50 \mathrm{MHz}$ oscillator installed on DE0-CV development board.

\subsubsection{User I/O modules}

Interaction between the user and the FPGA unit is processed by two modules. These modules monitor start/stop buttons and control board LEDs.
For the purpose of testing the system after minor or major changes in the architecture (customised for user needs), an internal simulator module has been implemented. The module provides fake sensor impulses of any frequency up to the capabilities of PLL component. One 'once per revolution' and three blade sensor signals can be simulated.

The FPGA unit requires four $3.3 \mathrm{~V}$ input signals provided to appropriate DE0-CV pins. Ethernet transmission is realized by two dedicated output pins. As the DE0-CV platform provides two 40-pin GPIO ports, the system can be easily adapted to monitor additional blade sensors. The FPGA unit is presented in Fig. 1.

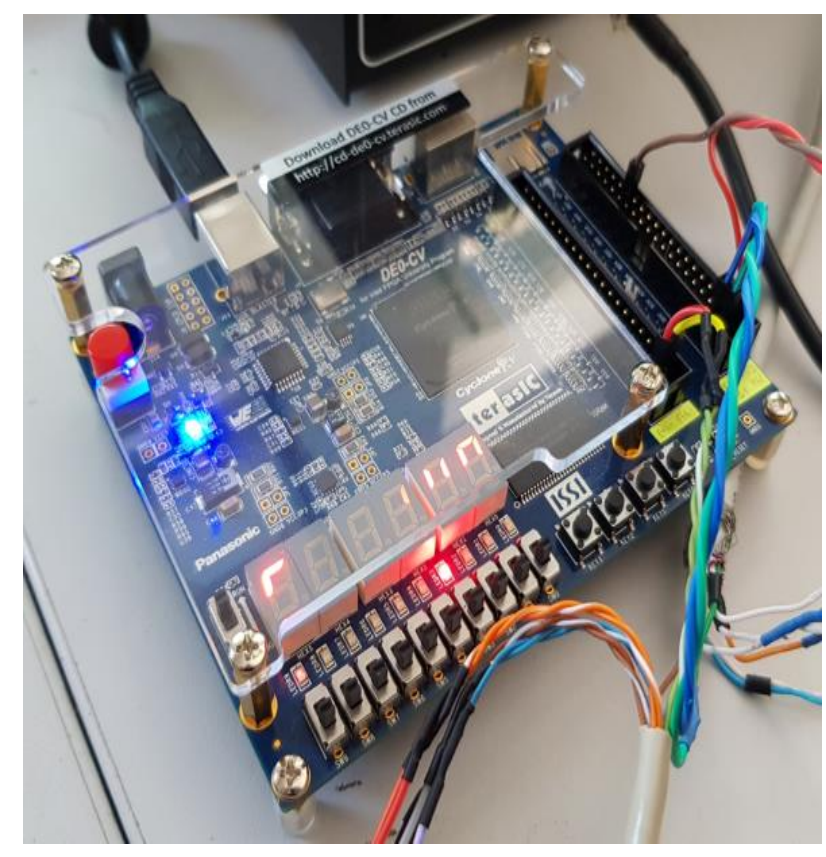

Fig. 1. FPGA unit - TerasIC DEO-CV development board.

\subsection{PC unit}

Apart from the FPGA data acquisition unit, the system includes a module run on a standard PC. The PC unit is a desktop application developed in C\# (.NET) in MS Visual Studio 2017 IDE. It requires a 64-bit MS Windows operating system and a computer with Ethernet RJ-45 interface. The application collects measured ToA values on 1024 port, de-serializes them, checks for transmission errors and saves the values in .csv files. An error is registered when two (or more) consecutive packets are not identified with appropriate rotation numbers (also included in data transmission). Application interface allows the user to start/stop data acquisition and to monitor the number of transmission errors, total measurement time and acquired data size. In order to avoid accidental data loss, it is saved in several .csv files, every 30 seconds of measurement. To improve the readability of data, each sensor has been assigned its own set of .csv files. The PC application window is presented in Fig. 2

\subsubsection{Internal simulator of sensor signals}




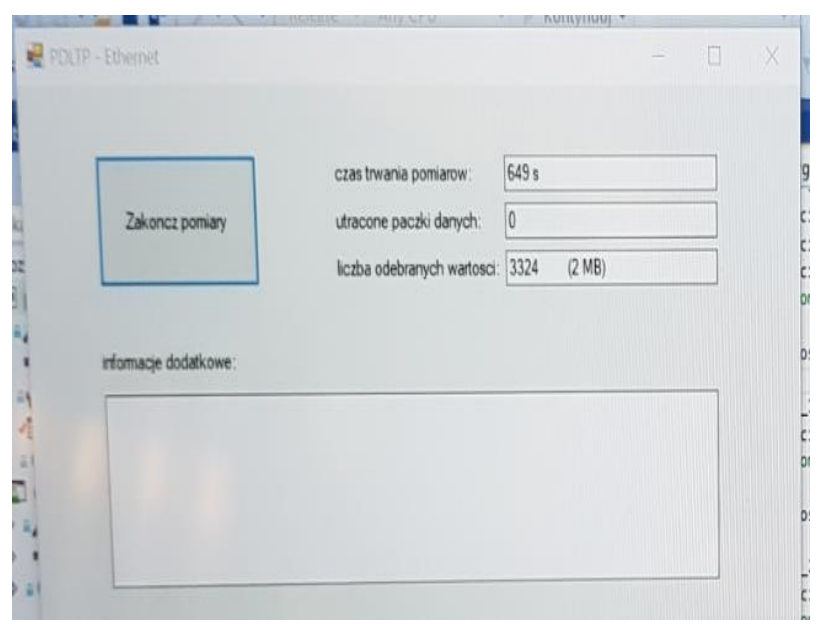

Fig. 2. PC unit - .NET desktop application for receiver PC.

\subsection{Measurement system set}

The system presented in this paper has been designed to perform measurements for various types of gas and steam turbine. Depending on the configuration of the sensors in turbine, the set of additional equipment for the system can vary. Tests made in a $380 \mathrm{MW}$ steam turbine in Bełchatów required to use optical-to-TTL converters in order to provide 3.3V impulses for system's inputs [6]. During the measurements conducted for an SO-3 jet engine turbine, input impulses were generated by an external converter module. Due to SO-3 test environment specifications (noise, safety), a fibre optic Ethernet connection with dedicated converters between FPGA and PC units was used. The distance between both system units in this case was up to $50 \mathrm{~m}$. Fig. 3 presents the basic system (FPGA and PC unit, PC USB-to-Ethernet adapter, a cable for the FPGA-PC connection and a cable connecting the FPGAunit with $3.3 \mathrm{~V}$ input signals coming from sensors).

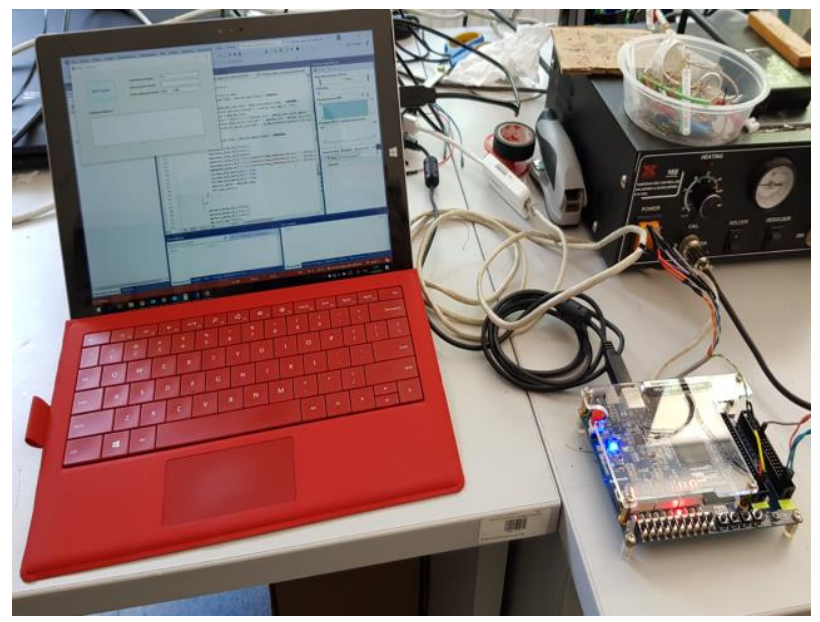

Fig. 3. B asic system: FPGA and PC units together with cables and adapters.

\subsection{Advantages of the system and future development}

Requirements for the system have been presented at the beginning of chapter 2 . Nevertheless, values for number of turbine blades, number of sensors and maximum frequency should not be treated as unchangeable. The biggest advantage of the system, apart from its now cost, is its flexibility. FPGA-architecture chips have been designed to perform high-speed data processing. Every effort has been undertaken to make the implementation of the system as efficient as possible, for which reason only $3 \%$ of the device's ALMs, $1 \%$ of block memory bits and $62 \%$ of the total number of $\mathrm{I} / \mathrm{O}$ pins have been used. The summary of the compilation for the system in Quartus Prime is presented in Fig. 4.

\begin{tabular}{|c|c|}
\hline \multicolumn{2}{|l|}{ Flow Summary } \\
\hline Q «<Filter $\rangle$ & \\
\hline Flow Status & Successful - Mon Aug 26 14:59:29 2019 \\
\hline Quartus Prime Version & 18.1.0 Build 625 09/12/2018 SJ Lite Edition \\
\hline Revision Name & Pomiary_czasow \\
\hline Top-level Entity Name & Pomiary_czasow_schemat \\
\hline Family & Cyclone V \\
\hline Device & 5CEBA4F23C7 \\
\hline Timing Models & Final \\
\hline Logic utilization (in ALMs) & $504 / 18,480(3 \%)$ \\
\hline Total registers & 761 \\
\hline Total pins & $138 / 224(62 \%)$ \\
\hline Total virtual pins & 0 \\
\hline Total block memory bits & $32,768 / 3,153,920(1 \%)$ \\
\hline Total DSP Blocks & $0 / 66(0 \%)$ \\
\hline Total HSSI RX PCSs & 0 \\
\hline Total HSSI PMA RX Deserializers & 0 \\
\hline Total HSSI TX PCSs & 0 \\
\hline Total HSSI PMA TX Serializers & 0 \\
\hline Total PLLs & $1 / 4(25 \%)$ \\
\hline Total DLLs & $0 / 4(0 \%)$ \\
\hline
\end{tabular}

Fig. 4. Compilation data for system implemented in Cyclone $V$ 5CEBA 4F 23C 7 FPGA device.

The number of $62 \% \mathrm{I} / \mathrm{O}$ pins used is in fact inflated, as only $3 \%$ ( 8 pins in total) are needed for measurement and data transmission tasks. The rest of the pins were used to visualize the state of the system by controlling LEDs, and should be treated as non-essential to the system. The fact that the system uses so little of the resources in the device indicates great potential for future development. What is more, the design of the system was prepared by programming and connecting block modules, making the solution highly scalable. Only minor changes in the system are necessary in order to add processing of signals from additional turbine sensors. All that is required to monitor the ToA of more than 75 turbine blades is an increase in the size of FIFO buffer and changes in the constant values of less than 10 lines of source code of one module block.

Apart from system's flexibility, another (and maybe the most important for the user) feature of the system is its total cost. TerasIC DE0-CV, as COTS hardware is an easily accessible development board and costs about $\$ 150$. If the end user uses their own PC, they will only need a few additional items, such as cables, Ethernet adapter (if necessary) and a turbine-specific converter to the TTL 3.3V signal voltage level. Hence, the total cost of the system should be lower than $\$ 200$, which is about 50 times cheaper than the price of other comparable systems from vendors like ex. National Instruments. What is more, the system can be implemented without buying any 
commercial licences for the IDE/software environment like LabVIEW or Matlab.

Future development of the system includes its implementation in the System on Chip (SoC) FPGA device. TerasIC DE1-SoC platform [12] has already been chosen. The core chip of the board comprises two modules: FPGA (Cyclone V) and an ARM-based (CortexA9) microcontroller. In addition to the standard functionality provided by FPGA devices, it gives the user the possibility of implementing more complex functions, as the ARM-based module can be controlled by a source code developed in higher-level (than VHDL) programming languages, such as $\mathrm{C}$ or $\mathrm{C}++$. The idea is to implement in the FPGA all the modules responsible for ToA measurements, while all components for data transmission are included in the ARM microcontroller. TCP protocol is planned to be implemented. The user will also gain the possibility of remotely controlling the system via the Internet.

\section{Measurements results}

\subsection{Testing the system with simulated impulses}

Before testing the system on real engine turbines, three phases of tests were conducted. A brief description of each test is presented below.

\subsubsection{Tests with internal signal simulator}

As was described in 2.1.6, the design of the system includes an internal simulator module. Fake impulses simulate sensor signals. The data generated during the tests proved that system works correctly. In the internal simulator module blade vibrations do not exist.

\subsubsection{Tests with external signal generators}

Two external signal generators were used in the experiment. Four signals were generated in total: one 'once per rotation' signal and three independent signals simulating blade sensor impulses. The workbench for the measurements is presented in Fig. 5.

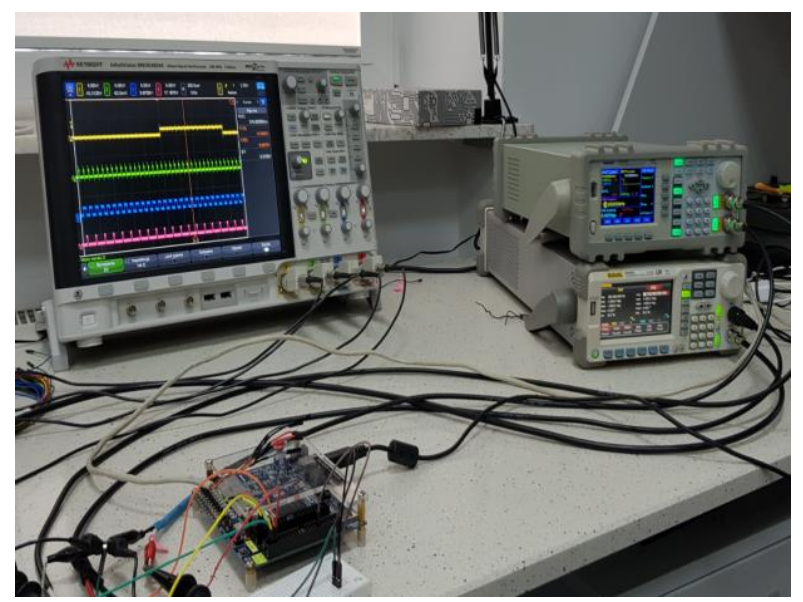

Fig. 5. Testing the system with external signal generators.

The signals were monitored on an oscilloscope. The measurement data was correct, although, as in 3.1.1, also in this case, no ToA deviations could be noted as blade vibrations do not exist.

\subsubsection{Tests with turbine simulator}

The third stage of system test was based on a turbine simulator developed at the Air Force Institute of Technology (AFIT) in Warsaw. It simulates signals from three sensors: one 'once per revolution' and two blade sensors (Fig. 7). No vibrations were registered, because the blades were simulated as a rectangular metal inserted into a plastic cylinder. The measurements workbench is presented in Fig. 6.

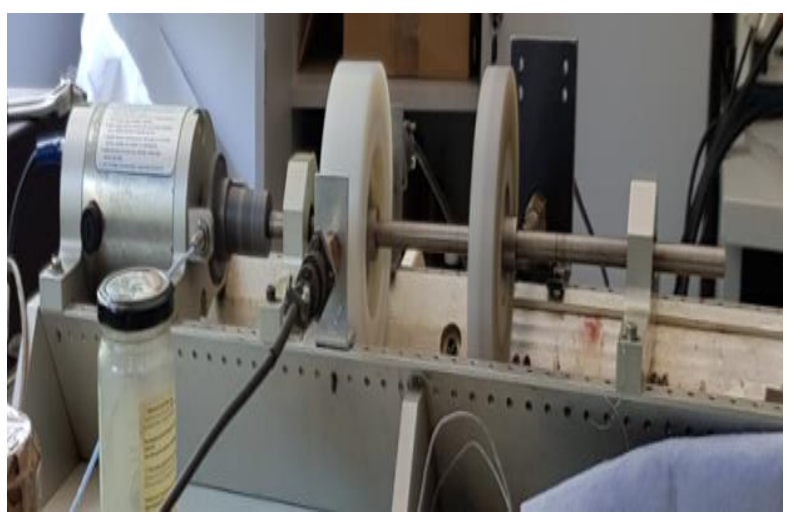

Fig. 6. Testing the system with turbine simulator at A FIT.

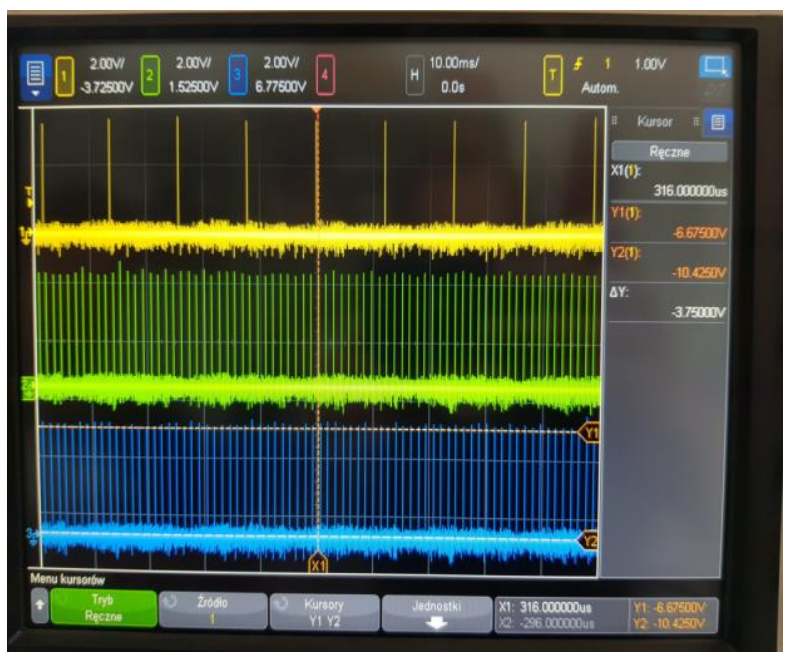

Fig. 7. Signals generated by turbine simulator: 'once per revolution' (yellow) and two blade sensor impulses (green and blue).

The simulator allows the user to change cylinder rotation speed and this feature was used when testing the system. The analysis of ToA data gathered during the test proved that system meets the design requirements.

\subsection{SO-3 jet engine}

Vibration measurements for an SO-3 jet engine turbine were made after getting the positive results described in 3.1.1, 3.1.2 and 3.1.3. Impulses were generated by 4 sensors in total (one 'once per revolution' and three blade sensors). A dedicated analogue-to-TTL $3.3 \mathrm{~V}$ converter was used for system inputs. The SO-3 engine measurements workbench is presented in Fig. 9. 
Vibrations were noted for each sensor. A fragment of a .csv file opened in MS Excel is presented in Fig. 9. The first column is a set of rotation identification numbers, the second (TW) is a set of ToA values for the 'once per rotation' sensor and the remaining columns present the ToA for subsequent turbine blades. It should be noted that the ToA for each successive blade increases.

\begin{tabular}{|c|c|c|c|c|c|c|c|c|}
\hline 4 & A & B & C & D & $E$ & $\mathrm{~F}$ & G & $\mathrm{H}$ \\
\hline 1 & nr_obr & TW & TB1_1 & TB1_2 & TB1_3 & TB1_4 & TB1_5 & TB1_6 \\
\hline 2 & 67720 & 39992 & 1203 & 2627 & 4047 & 5478 & 6901 & 8333 \\
\hline 3 & 67721 & | 39988 & 1203 & 2627 & 4047 & 5479 & 6901 & 8334 \\
\hline 4 & 67722 & 39991 & 1203 & 2625 & 4046 & 5478 & 6901 & 8333 \\
\hline 5 & 67723 & 39985 & 1203 & 2626 & 4046 & 5478 & 6901 & 8332 \\
\hline 6 & 67724 & 39989 & 1204 & 2627 & 4047 & 5478 & 6901 & 8333 \\
\hline 7 & 67725 & 39987 & 1203 & 2627 & 4047 & 5479 & 6901 & 8333 \\
\hline 8 & 67726 & 539991 & 1204 & 2627 & 4047 & 5479 & 6901 & 8333 \\
\hline 9 & 67727 & 39988 & 1203 & 2626 & 4046 & 5479 & 6900 & 8333 \\
\hline
\end{tabular}

Fig. 8. ToA measurement results in .csv file.

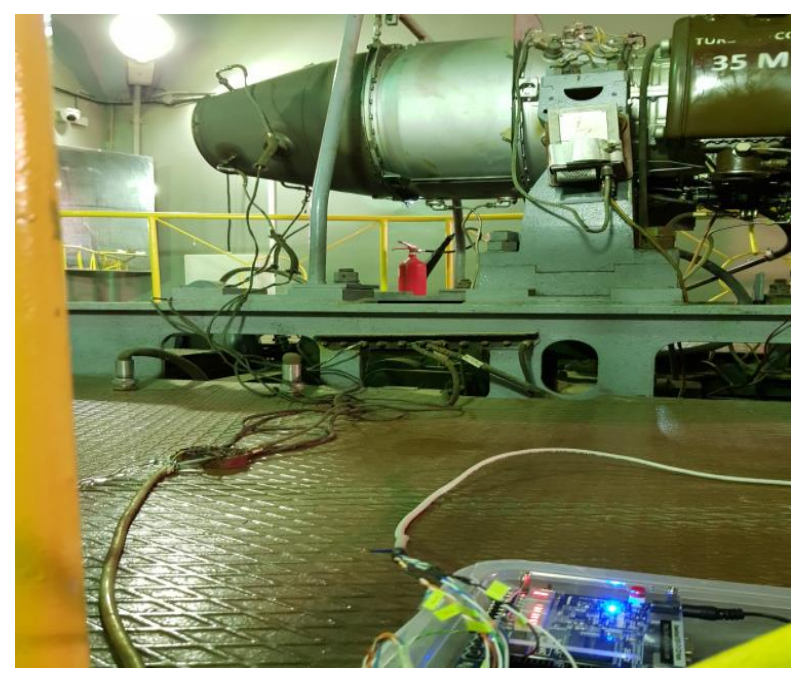

Fig. 9. S0-3 jet engine on stand.

Fig. 10 and Fig. 11 present all rotor blades vibrations. The maximal amplitude of $2.5 \mathrm{~mm}$ is in $42 \mathrm{~s}$ for rotating stall. The region of stall is from $0-42 \mathrm{~s}$ (Fig. 10). The second region of maximal amplitude, from (105 - $120 \mathrm{~s}$ ) with maximum $1.5 \mathrm{~mm}$ at $115 \mathrm{~s}$, is connected with $2 \mathrm{EO}$ excitation [5].

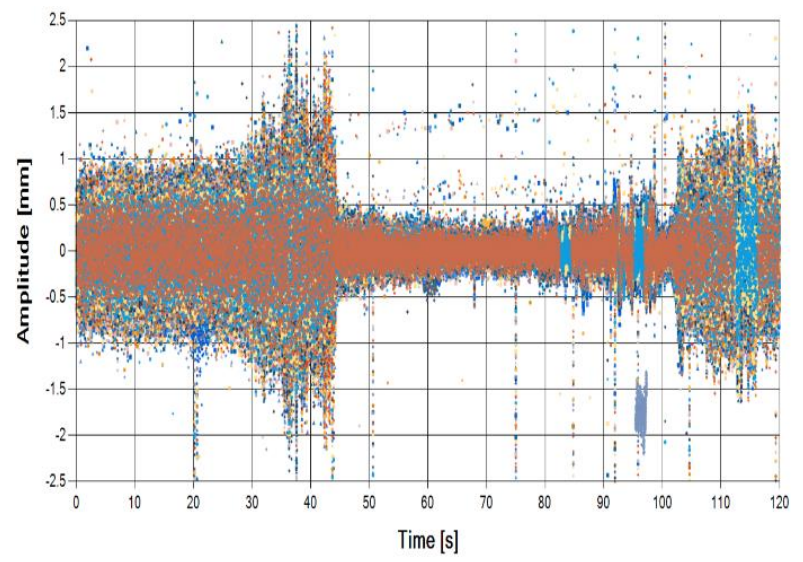

Fig. 10. A II rotor blade amplitude of the first stage of $S O-3$ engine

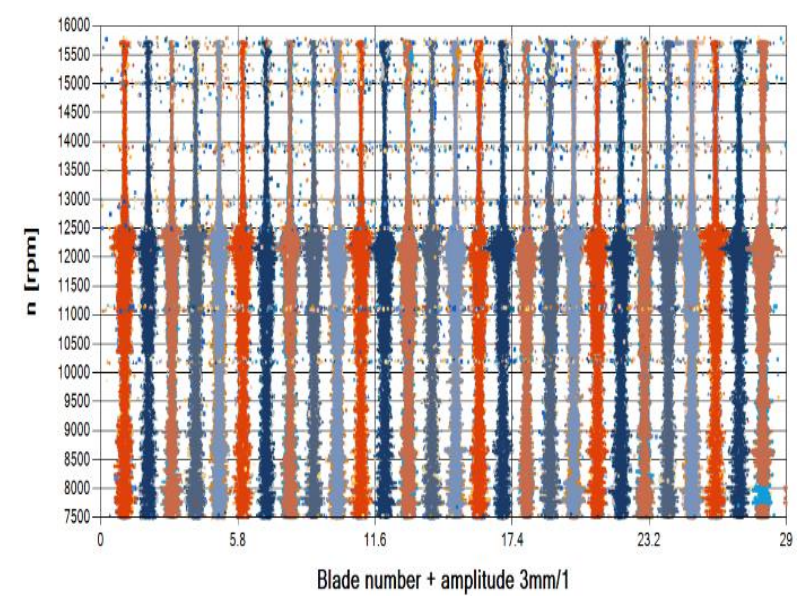

Fig. 11. Separate rotor blade amplitudes of the first stage of so-3 engine

\section{Conclusions}

The system described in this paper is effective at $15000 \mathrm{rpm}$. Its main advantage, a cost of less than $\$ 200$, sets it apart from other comparable solutions. The system was thoroughly tested before making blade vibration measurements of an SO-3 jet engine compressor. The analysis of the results proved its usability as the vibrations were accurately detected and measured. The architecture of the system is highly scalable and suitable for further developments, such as, using additional sensors, changing rotation speeds, and allowing the user to control the system remotely. No packets were lost while transmitting data from the FPGA module to the PC unit during the tests and final measurements. Measurements of rotor blade amplitude in the first stage of the SO-3 engine using the new system were similar to those of other systems and numerical results.

\section{References}

[1] H. S. Dhadwal i A. P. Kurkov, „Simultaneous Optical Measurements of Axial and Tangential Steady-State Blade Deflections," NASA TM1999-209051, (1999).

[2] Y. Zablotskiy, A. K orostelev i L. Sviblow, „Device for Non-Contact A nalysis of V ibration Movement of Turbomachinery Blades," Biuletyn, Otkrytia, izobretenija, promyszlennyje obrazcy, tovarnyje znaki, A vt. Svid. No 160886, no 5, (1964).

[3] M. Zielinski, ,MTU Perspective on Tip-Timing, Panel Session, Tip-Timing: State-of-the-A rt and Future Developments," ASME TURBO-EXPO, Denmark, Copenhagen, GT2012-70184, J une 11-15, (2012).

[4] P. Russhard, ,Perspective on Tip-Timing, Panel Session, Tip-Timing: State-of-the-A rt and Future Developments," ASME TURBO-EXPO, Denmark, Copenhagen, June 11-15, (2012).

[5] R. Szczepanik, Rzadkowski R.Dynamic Experimental A nalysis of Dynamic Properties of A ircraft Engine Rotor B lades in V arious 
Operating Conditions," PBI Radom, (2012) in Polish.

[6] R. Przysowa, „Blade Vibration Monitoring in a L ow-Pressure Steam Turbine," ASME TURBOEXPO: Power for Land, Sea and Air, V olume 6: Ceramics; Controls, Diagnostics and Instrumentation; Education; $M$ anufacturing $M$ aterials and M etallurgy ():V 006T 05A 025, pp. 1-11, (2018).

[7] Altera, DE 1 Development and Education Board U ser M anual, A Itera Corporation, (2006).

[8] Altera, Cyclone II Device Handbook, V olume I, Altera Corporation, (2008).

[9] TerasIC, DEo-CV U ser M anual, Terasic Inc., (2015).

[10] Altera, Cyclone V Device Handbook,, A Itera Corporation, (2019).

[11] Intel, „Intel FPGA IP Portfolio,” Intel, (2019). [Online]. A vailable: https://www.intel.com/content/www/us/en/produ cts/programmable/intellectual-property.html. (2019).

[12] TerasIC, DE1-SoC U ser M anual, TerasIC, (2015). 